\title{
A Multi-Antenna Capability for the EISCAT Svalbard Radar
}

\author{
P. J. S. WiLLIAMS \\ Adran Ffiseg, Prifysgol Cymru Aberystwyth, SY23 3BZ, Wales \\ (Received February 13, 1995; Revised August 20, 1995; Accepted August 22, 1995)

\begin{abstract}
The case for a multi-antenna capability to serve the EISCAT Svalbard radar is presented under three headings: a) the need to make reliable measurements of plasma velocity, b) the ability to operate with increased sensitivity when electron concentrations are low and c) the flexibility to employ several different modes during an experiment.
\end{abstract}

\section{Introduction}

The original proposal for an incoherent-scatter radar on Svalbard $\left(78.1^{\circ} \mathrm{N}, 16.0^{\circ} \mathrm{E}\right)$ recommended a system of three antennas fed by a transmitter with $3 \mathrm{MW}$ peak power (van Eyken et al., 1989). This recommendation was endorsed by the Working Group set up by EISCAT Council to provide a design specification for the radar (Bjørna et al., 1991). The first phase of the EISCAT Svalbard Radar (ESR), as approved by EISCAT Council, consists of a single antenna with a $0.5 \mathrm{MW}$ transmitter operating at 500 $\mathrm{MHz}$. However the long-term aim remains a three antenna system, with a transmitter of at least $1.5 \mathrm{MW}$ peak power, and in considering the next phase of development the advantages of two antennas are so great that the top priority must be the addition of a second antenna.

In the first place, a multi-antenna system is necessary to make reliable measurements of plasma velocity in the very dynamic environment of the polar cap. To estimate the full vector of plasma velocity it is necessary to measure the components of velocity in three non-planar directions. In the past this has been attempted in three different ways. In the monostatic, beam-swinging method a single antenna swings through three directions in sequence. However, this method is only valid if the plasma velocity is constant over the time taken to complete the cycle and over the horizontal area covered in the scan. In the tristatic method three spaced antennas make simultaneous measurements of the echoes scattered from a single volume. This method gives a true unbiased estimate of the velocity vector but it requires three sites at a suitable spacing, and the vector suffers from relatively large random errors. The third method employs a multi-beam radar to make continuous and simultaneous measurements in different directions; it is therefore able to monitor and correct for temporal and spatial variations. This is the method recommended for the ESR.

Once extra antennas are constructed they will offer several other advantages. For example, if two (or three) antennas are closely spaced it will be possible to point all three in the same direction and operate them in phase to provide a single-beam radar with a proportional increase in collecting area. Finally, the opportunity to use different multi-beam and single beam modes in sequence provides a very valuable flexibility in designing experiments for an environment where sudden changes between high and low electron concentration and between quiet and very active auroral conditions can be anticipated.

The case for a multi-antenna system can therefore be argued under three headings:

a) measurements of plasma velocity,

b) measurements with high sensitivity,

c) flexibility to choose from a wide range of operating modes. 


\section{Measurements of Plasma Velocity}

\subsection{Advantages and disadvantages of different methods of measurement}

The vector of $F$-region plasma velocity is one of the most important parameters measured by incoherent scatter. From this parameter it is possible to derive the corresponding electric field, which is the ionospheric signature of magnetospheric convection and also the key parameter controlling the Hall and Pedersen currents. Each method of measuring plasma velocity has its advantages and disadvantages.

i) The monostatic beam-swinging method

The essence of this method is that a single antenna swings through three non-planar directions in sequence. Measurements in each direction are integrated for the dwell-time necessary to provide a component of plasma velocity with small-enough random error before slewing to the next direction. The cycle of three directions is repeated and the three components are combined to give the full vector of velocity, using interpolation to ensure that all components correspond to the same equivalent time.

Variations of the basic method involve continuous scanning, or scanning through a larger number of directions, but the basic assumptions of all beam-swinging experiments are the same: the drift velocity of the plasma is assumed constant - or changing steadily — over the time taken to complete a single cycle of the scanning pattern and constant over the horizontal distances separating the three scattering volumes.

This is the only method possible when the radar is restricted to a single, steerable antenna, and it has been used extensively in incoherent-scatter radars such as those at Millstone Hill, Arecibo, and Chatanika/ Søndrestrømfjord. It has also been used at EISCAT, where observations from the four positions of common programme CP2 have been combined to derive the ion velocity in both the $F$ region (Williams et al., 1984) and the $E$ region (Williams et al., 1994). In addition, measurements made in the two positions of common programme CP4, and the UK special-programme POLA, have been combined to derive the $F$-region plasma velocity at high latitudes (e.g. van Eyken et al., 1984). Beam-swinging will be the only way for the ESR to measure plasma velocities until a second antenna is built.

A monostatic system with a single antenna is clearly the cheapest option for an incoherent-scatter radar. Such a system makes its measurements over an extensive range of heights: for example the EISCAT uhf system typically measures ionospheric parameters over a height range stretching from below $100 \mathrm{~km}$ to over $600 \mathrm{~km}$. These measurements are made with a range resolution $\delta R$ which is defined by the length of the transmitted pulse $\tau(\delta R=1 / 2 c \tau)$ and for measurements in the $F$ region and topside $\tau$ can be $360 \mu \mathrm{s}$ or even greater without the loss of height resolution having any serious effect on the quality of the results.

A monostatic radar also provides high sensitivity. Let an antenna of effective collecting area $A_{\mathrm{c}}$ and loss-factor $L$ transmit a pulse of peak-power $P$ and length $\tau$. At range $R$ this illuminates a volume of the ionosphere with electron concentration $N$ and ion and electron temperatures $T_{\mathrm{i}}$ and $T_{\mathrm{c}}$ respectively. The echoes from this volume, spread over $a$ bandwidth $b$, are received by the same antenna and delivered to a receiver with a system temperature $T_{\text {sys. }}$. For all wavelengths much greater than the Debye length, the total bandwidth of the scattered signal is given by:

$$
b \cong 4.8 \cdot \sqrt{\frac{8 k_{\mathrm{B}} T_{\mathrm{i}}}{m_{\mathrm{i}} \lambda^{2}}}
$$

and the ratio of the signal power in the echo $P_{\mathrm{s}}$ to the background noise power $P_{\mathrm{n}}$ over the same bandwidth $b$ is given by:

$$
\frac{P_{\mathrm{s}}}{P_{\mathrm{n}}}=\frac{34.8 L^{2} P A_{\mathrm{c}} \tau}{R^{2} b T_{\mathrm{sys}}} \cdot \frac{N}{1+T_{\mathrm{e}} / T_{\mathrm{i}}}
$$


(Rishbeth and Williams, 1985).

For a single 32-m dish with an aperture efficiency of $73 \%$, a loss factor of 0.86 and a system noise temperature of $80 \mathrm{~K}$, transmitting a peak power of $0.5 \mathrm{MW}$ at a frequency of $500 \mathrm{MHz}$ (the parameters of phase 1 of the ESR), the echo from the $F$ region offers a signal-to-noise ratio:

$$
S N R=\frac{P_{\mathrm{s}}}{P_{\mathrm{n}}} \approx \frac{0.18 \times 10^{6} \tau}{R^{2}} \cdot \frac{N}{\sqrt{T_{\mathrm{i}}}\left(1+T_{\mathrm{e}} / T_{\mathrm{i}}\right)} .
$$

To measure a component of plasma velocity the pulse is repeated at a rate $n$ and the echoes from a given height are integrated for time $t$. The random noise error in the measurement is then given to a good approximation by the formula (Jones et al., 1986):

$$
\delta v=\frac{2.4 T_{\mathrm{i}}^{0.25}\left(0.5+S N R^{-1}\right)}{(n \tau t)^{0.5}}
$$

As a typical example, for $N=10^{11} \mathrm{~m}^{-3}$ and $T_{\mathrm{i}}=1600 \mathrm{~K}$ the $S N R=0.5$. If the full duty cycle $(n \tau)$ of $25 \%$ is used for the velocity measurement and the results are integrated for 1 min then $\delta v \approx 10 \mathrm{~m} \mathrm{~s}^{-1}$.

Moreover, assuming that the magnetic field lines are at constant potential the monostatic measurements of $F$-region plasma velocity made at different heights along the same magnetic field line can be combined to give an overall estimate of the electric field strength with even smaller random errors (Jones et al., 1986).

The main disadvantage of a monostatic system is that if there are temporal or spatial variations in plasma velocity on a scale comparable with, or smaller than, that of the scanning pattern then systematic errors are introduced. The nature of these systematic errors is sometimes misunderstood.

It is obvious that a beam-scanning method applies a low-pass filter to the data. If, for example, a beam-scanning programme takes 3 minutes to complete a cycle and follows a horizontal circle of radius $100 \mathrm{~km}$ then it cannot record events that change significantly on a time scale much less than 3 minutes, or occur on a spatial scale much less than $100 \mathrm{~km}$. However, it is sometimes assumed that the method gives a valid estimate of the plasma velocity when averaged over the cycle time and the area covered. This is not the case. If velocity components affected by temporal or spatial changes are combincd to generate a full velocity vector these changes generate spurious velocities by the process described as "mixing" (Etemadi et al., 1989).

To illustrate this in a simple two-dimensional case, Fig. 1 shows how two line-of-sight components of velocity $v_{1}$ and $v_{2}$ are combined to generate a single vector represented by the perpendicular components $v_{x}$ and $v_{y}$. If the true vectors in the two scattering volumes are $v_{x, 1}, v_{y, 1}$ and $v_{x, 2}, v_{y, 2}$ then:

$$
v_{1}=v_{y, 1} \cos \theta-v_{x, 1} \sin \theta \text { and } v_{2}=v_{y, 2} \cos \theta-v_{x, 2} \sin \theta
$$

so that the estimate of velocity derived from $v_{1}$ and $v_{2}$ is given by:

$$
\begin{gathered}
v_{x}=\frac{v_{2}-v_{1}}{2 \sin \theta}=\frac{v_{x, 1}+v_{x, 2}}{2}+\frac{\left(v_{y, 2}-v_{y, 1}\right) \cot \theta}{2} \\
=\left\langle v_{x}\right\rangle+\frac{\left(v_{y, 2}-v_{y, 1}\right) \cot \theta}{2}
\end{gathered}
$$



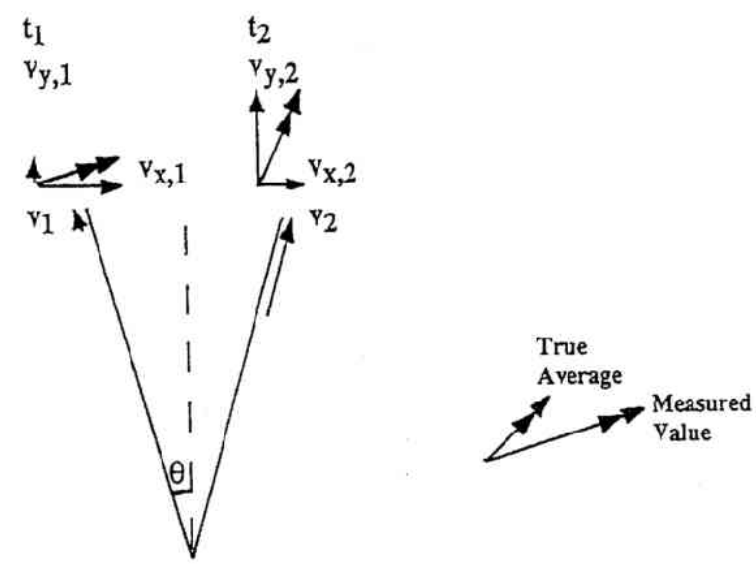

Fig. 1. The generation of systematic "mixing" errors by beam-swinging.

$$
\begin{gathered}
v_{y}=\frac{v_{2}+v_{1}}{2 \cos \theta}=\frac{v_{y, 1}+v_{y, 2}}{2}+\frac{\left(v_{x, 2}-v_{x, 1}\right) \tan \theta}{2} \\
=\left\langle v_{y}\right\rangle+\frac{\left(v_{x, 2}-v_{x, 1}\right) \tan \theta}{2} .
\end{gathered}
$$

The second term in Eqs. 5(a) and 5(b) represents the systematic "mixing" error. For relatively small values of $\theta$ (c.g. $=11.2^{\circ}$ in EISCAT common programme CP4) the largest error appears in the $x$-component, perpendicular to the direction bisecting the two pointing directions. This is sometimes obvious in the results from beam-swinging experiments where the vectors of plasma velocity appear to follow a circle centred on the radar site.

\section{ii) The tristatic method}

The tristatic method makes simultaneous measurements from three separate antennas, set at the apices of a triangle with sides of $100 \mathrm{~km}$ or more, all looking into the same scattering volume. Each antenna can determine the component of velocity along the appropriate mirror direction i.e. the direction bisecting the angle between the beams of the transmitting and receiving antennas.

The UK incoherent-scatter system was the first to employ this method, transmitting a cw wave vertically upwards from Malvem and receiving the scattered signals at Wardle, Aberystwyth and Chilbolton (Rishbeth et al., 1973). Shortly afterwards a similar but more powerful system was inaugurated in France with an antenna at St. Santin transmitting a cw wave vertically upwards and antennas at Nancay, Monpazier and Mende receiving the scattered signal (Bauer et al., 1974).

The EISCAT radar marks a major advance in the method: a pulsed signal is transmitted from Tromsø and the scattered echoes are received at Tromsø itself, at Kiruna and at Sodankylä. By using a pulsed transmission, the system at Tromsø is able to measure the profiles of scalar parameters over a range of heights, while the compression of transmitted power into a pulse, so that the remote sites need only receive signals for the precise interval when the pulse is in the beam, increases the accuracy of the measurements for a given average power.

A tristatic radar has the advantage that all three components of plasma velocity are measured simultaneously from the same volume of plasma and so it is not necessary to assume that the plasma velocity is constant in space or time.

The obvious disadvantage of a full tristatic system is that it is necessary to build and operate three receiving stations and this adds substantially to the overall cost. In addition, measurements of a velocity 
vector by a tristatic radar usually suffer larger noise errors than measurements from a monostatic radar. The scattering volume for echoes received at a remote site is defined by the intersection of the two antenna beams, and this is usually smaller than the effective scattering volume employed in monostatic measurements, which is defined by the pulse length. This handicap is only partly offset by the smaller system noise possible in a purely passive receiving system.

Let a bistatic radar at wavelength $\lambda$ operate at peak-power $P$ with two parabolic dishes of diameter $d$, aperture efficiency $\eta$ and loss-factor $L$ spaced a distance $D$ apart. If the transmitting antenna points vertically upwards and the two beams intersect at an angle $\gamma$, then the signal-to-noise ratio is given by:

$$
S N R=\frac{P_{\mathrm{s}}}{P_{\mathrm{n}}}=\frac{1.2 \times 10^{-9} \eta^{2} L^{2} P d \lambda^{2}\left(1-\sin ^{2}[\gamma] / 2\right)}{D T_{\mathrm{sys}} \cos (\gamma / 2)} \cdot \frac{N}{\sqrt{T_{\mathrm{i}}}\left(1+T_{\mathrm{e}} / T_{\mathrm{i}}\right)}
$$

and the error in the velocity measurement is given by

$$
\delta v=\frac{2.4 T_{\mathrm{i}}^{0.25}\left(0.5+S N R^{-1}\right)}{(\cos (\gamma / 2) n \tau t)^{0.5}}
$$

(note: this formula assumes that the transmitted pulse totally fills the intersection volume and so it breaks down for small values of $\gamma$ where the intersection range exceeds the pulse length).

One problem at Svalbard is that on the main island only a small number of sites, such as Hornsund or Ny Ålesund, would be suitable for building a remote station. Each of these sites is at a distance of $\sim 130$ $\mathrm{km}$ from the transmitting site near Longyearbyen so for velocity measurements in the $F$ region $\gamma \approx 23^{\circ}$. For a 32-m parabolic dish at one of these sites, with a system noise temperature of $40 \mathrm{~K}$ and assuming as before that $N=10^{11} \mathrm{~m}^{3}$ and $T_{\mathrm{i}}=1600 \mathrm{~K}, S N R=0.5$. It follows that if the full duty cycle of $25 \%$ is used for the velocity measurement and the results are integrated for $1 \mathrm{~min}, \delta v \approx 10 \mathrm{~m} \mathrm{~s}^{-1}$.

This error in a single component is comparable with the error in a single component measured by the monostatic method, but in the tristatic case the angles between the different components are usually much smaller than the angles chosen in the monostatic method, so when matrix multiplication is used to convert measured components into a full vector the exponents in the matrix are larger in the tristatic case. In addition, monostatic measurements can be made simultaneously for several gates in the $F$ region and as the noise errors in these measurements are independent, they can be added together with suitable weighting to reduce the random errors significantly.

The different advantages and disadvantages of the beam-swinging and tristatic methods of measuring plasma velocity are clearly illustrated in Fig. 2, taken from Farmer et al. (1990). These results are based on EISCAT measurements where both beam-swinging and tristatic methods were used simultaneously. In the observations before 2130 UT, the electron concentration was very low and the random noise errors are clearly larger in the tristatic measurements. At about 2130 UT, however, there was a sudden change in the pattern of plasma velocity accompanied by a marked improvement in the signal-to-noise ratio. The tristatic measurements now give reliable and consistent measurements, which display the sudden onset of a large eastward velocity. However, at the start of this period of rapidly-changing velocity (indicated by circles), the beam-swinging method gives an apparent westward velocity, in exactly the opposite direction. This illustrates a systematic "mixing" error. In this case the total set of beam-swinging measurements took 10 minutes and the physical separation of the two volumes whose velocity components were combined to give the total vector was between 200 and $300 \mathrm{~km}$ - sufficient separation in time and space for one volume to lie in a region of strong eastward velocity while the other was in a region where the velocity was much lower.

This problem is one which is aggravated as the observations move to higher magnetic latitude. Atmid 


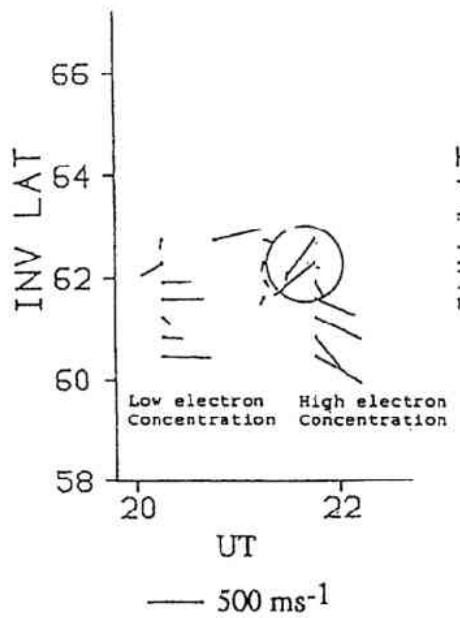

Beam-swing Velocities

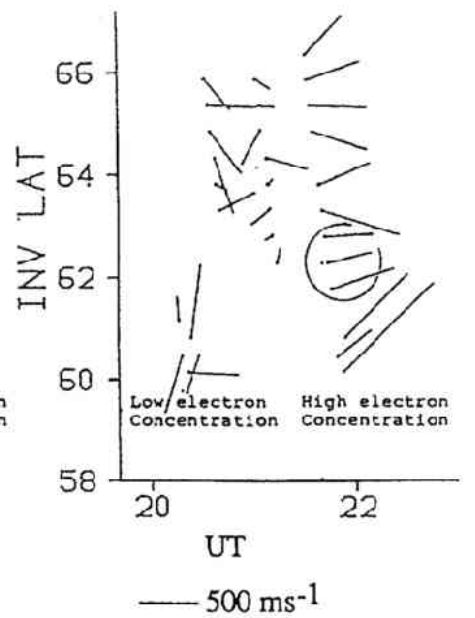

Tristatic Velocities

Fig. 2. Velocity vectors for 1986 September 11 comparing the vectors derived by a tristatic method with those derived from a monostatic beam-swinging method.

latitudes, time separations of a few minutes or spatial separations up to a few hundred kilometres are usually acceptable (e.g. Oliver et al., 1988) but at an invariant latitude of $80^{\circ}$ a separation of $300 \mathrm{~km}$ is equivalent to a difference of over 1 hour in MLT and in the polar cap such differences might correspond to entirely different regimes of plasma flow.

iii) The multi-beam method

The multi-beam method uses more than one antenna beam at a single site to look simultaneously in different directions. This method combines many of the advantages of the monostatic beam-swinging and tristatic methods. It only requires one site, and to build and operate two (or three) antennas at one site is much cheaper than to build and operate a single antenna at two (or three) sites. This method also shares with the single-beam mode the advantages of high signal-to-noise and the opportunity to combine independent estimates of plasma velocity made at different heights.

Moreover, in multi-antenna observations it is possible to distinguish temporal and spatial variations and the only necessary assumption is that either the plasma velocity is constant over the horizontal distances separating the three scattering volumes but may vary with time, or else a spatial pattern of plasma velocity remains constant as it drifts through the three scattering volumes.

This method is already in use at EISCAT where the common programme $\mathrm{CP} 4$, which had originally used beam-swinging, now operates the vhf radar in split-beam mode to provide two beams observing simultaneously $14.5^{\circ}$ apart. More recently the common programme CP6 has also adopted a split-beam mode to provide velocities in the mesosphere and lower thermosphere. An equivalent method is used by the MU radar in Japan. In this case the antenna system is a phased array and electronic beam-swinging is so rapid that measurements made by interlacing observations from different directions can be regarded as simultaneous (Oliver et al., 1988).

It follows that in deciding the optimum mode for measurements in the auroral zone or polar cap the crucial questions are whether the $F$-region plasma velocity can be assumed constant over times of 3 minutes or over distances of $100 \mathrm{~km}$. EISCAT measurements have demonstrated that during active periods neither of these assumptions can be made.

For example, EISCAT tristatic measurements of plasma velocity in a single volume show that the electric field is dominated by short-lived bursts. (Williams et al. 1990a, 1992; Lewis et al., 1992, 1994). Whereas the overall duration of such a flow burst is typically 5-10 minutes, the actual electric field strength 
at a given point has been seen to increase from $50 \mathrm{mV} \mathrm{m}^{-1}$ to $180 \mathrm{mV} \mathrm{m}^{-1}$ in just over $1 \mathrm{~min}$ and fall just as rapidly (Williams et al., 1990b). Indeed, in January 1993 EISCAT measured an electric field of well over $200 \mathrm{mV} \mathrm{m}^{-1}$ which lasted for less than $10 \mathrm{sec}$ (Lanchester et al., 1995). For events such as these a monostatic single-beam radar would certainly give spurious results.

However, there is also a serious limitation in tristatic measurements of flow-burst events. Although the measurements give an unbiased estimate of the time variation of electric field for a single volume they cannot distinguish between a short-lived plasma flow burst occurring simultaneously over a wide area surrounding the beam, a sharp boundary between regions of high and low plasma velocity oscillating toand-fro through the beam, or a long-lived, narrow band of enhanced plasma velocity drifting through the beam. There is evidence that all three patterns occur (Williams et al., 1992).

On some occasions simultaneous measurements by EISCAT and an auroral radar such as STARE indicate a short-lived flow burst covering several degrees of latitude and longitude (Williams et al., 1992). At other times, records of an all-sky camera indicate the zonal drift of an omega band so that the boundary between the regions of low and high electric field crosses and recrosses the EISCAT beam (Luhr and Schlegel, 1994). Finally, flow-bursts have been identified with a band of enhanced electric field on one edge of an auroral arc which drifts meridionally through the EISCAT beam (Opgenoorth et al., 1990). Measurements of the associated ion frictional heating suggest that such bands are $40 \mathrm{~km}$ or so in width (Lewis et al., 1994).

The advantages of a multi-beam facility in observing phenomena like these can be demonstrated by simulating the response of a single-beam and a multi-beam system to different patterns of plasma velocity and comparing the results derived.

\subsection{Simulation of measurements made by single-beam and multi-beam radars}

To compare the reliability of beam-swinging and multi-beam measurements from a single site, observations using both methods were simulated in a computer model. Two simple patterns of plasma velocity were used in this simulation, both corresponding to phenomena that will be studied by the ESR, and in both cases it was assumed that the pattern drifted steadily across the three scattering volumes of the radar.

In the first case, the pattern describes a reversal of electric field/plasma velocity at a boundary. For example, in all convection patterns associated with southward-pointing IMF there are flow reversals between the generally antisunward velocities observed in the polar cap and the generally sunward flows of the auroral oval, and the boundary separating such flow reversals may expand or contract according to the rate of reconnection on the dayside and nightside (e.g. Lockwood 1991a, b). Especially important in this aspect of the work of the ESR will be studies of flow velocities in the region of the Cusp (see, for example, Fig. 3 in Cowley et al., 1990).

A similar pattern would describe regions of high and low plasma velocity, separated by a sharp boundary - a configuration which describes the change in plasma velocity at the boundary of an omega band (Luhr and Schlegel, 1994).

The second pattern simulates an auroral arc drifting steadily through the beam. The enhanced electron concentration associated with the arc forms a band of high conductivity and low electric field, assumed to be $10 \mathrm{~km}$ wide. Adjacent to the leading edge of the arc there is a parallel band, $20 \mathrm{~km}$ wide, where the electron concentration is reduced but the electric field strength is enhanced in order to maintain the Pedersen current flowing perpendicularly out of the arc. This pattern is preceded and followed by regions where the background clectric field applies. EISCAT has made a major contribution to the study of ares in the auroral zone (Opgenoorth et al., 1990; Lewis et al., 1994; Lanchester et al., 1995). A similar study of dayside aurorae and theta aurorae will form a vital part of the programme of the ESR.

In simulating a beam-swinging experiment a single antenna was scanned in a three-minute cycle through three directions splayed symmetrically about the local magnetic field to an angle of $15^{\circ}$. For each direction in turn interpolation was used to provide estimates of the corresponding component of plasma velocity at 1 -min intervals and three simultaneous components were then combined to give the full vector 
Monostatic Beam-swinging Method

a) Zonal Velocity

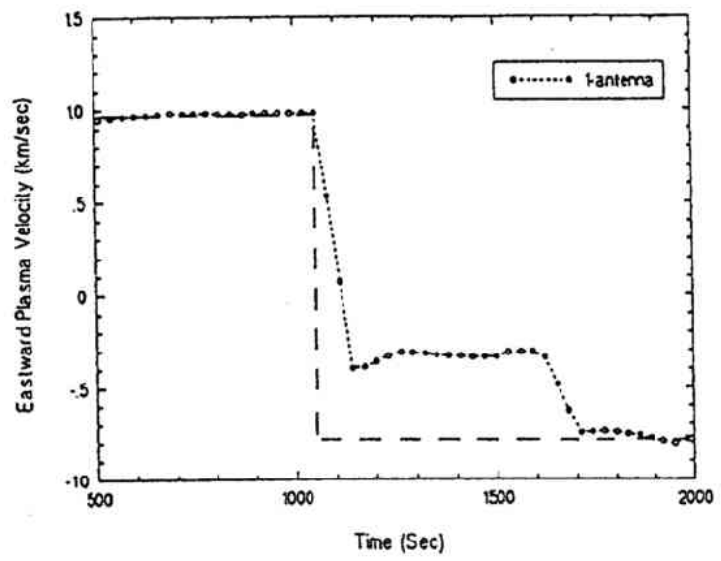

Monostatic Multibeam Method

c) Zonal Velocity

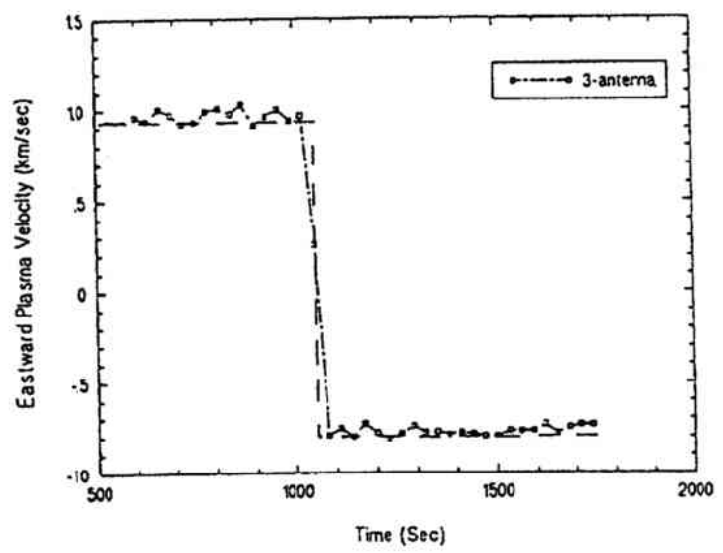

b) Meridional Velocity

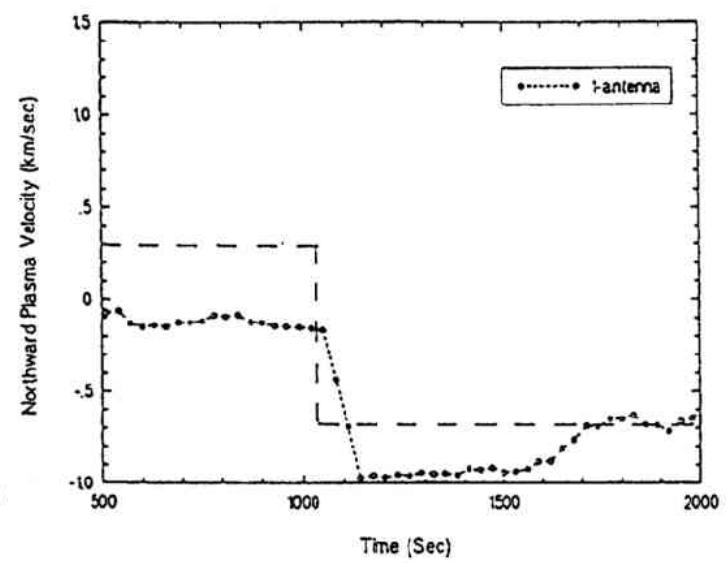

d) Meridional Velocity

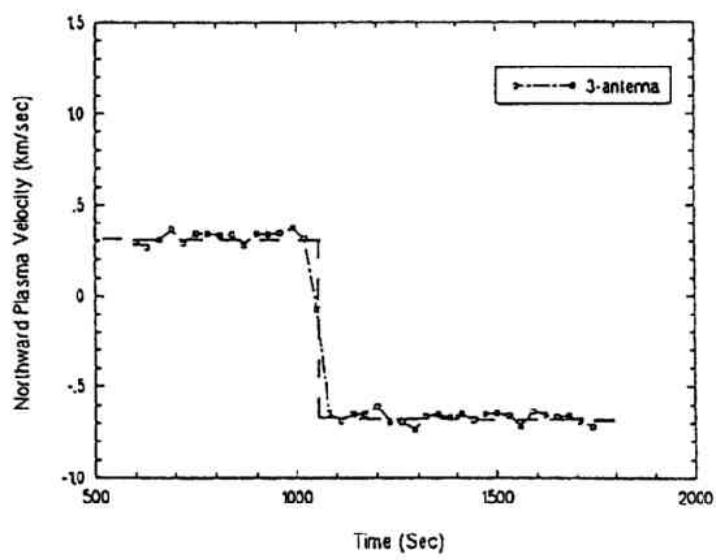

Model Value

Fig. 3. A comparison of simulated results obtained by a monostatic single-antenna beam-swinging method and a monostatic three-antenna multibeam method when observing the reversal of plasma velocity at a boundary which is drifting over the radar.

of plasma velocity.

In the multi-beam experiment three separate antennas made continuous measurements in the same three directions. In this case no interpolation was necessary and the time variation of the velocity components measured by the three antennas could be compared directly. If the spatial variation of plasma velocity follows a fixed pattern as it drifts through the three beams, cross-correlation of the three velocity components can be used to determine the drift velocity, and then appropriate time-shifts can be applied to the three components before they are combined to give the full vector. 


\section{Monostatic Beam-swinging Method}

a) Zonal Velocity

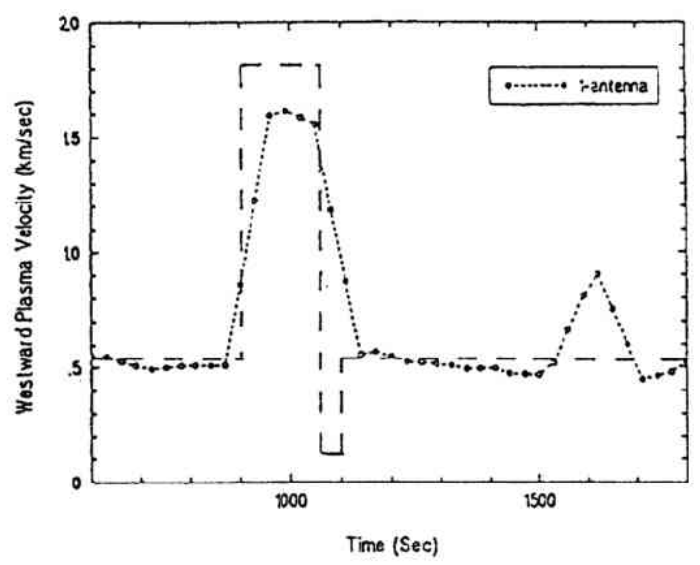

Monostatic Multibeam Method

c) Zonal Velocity

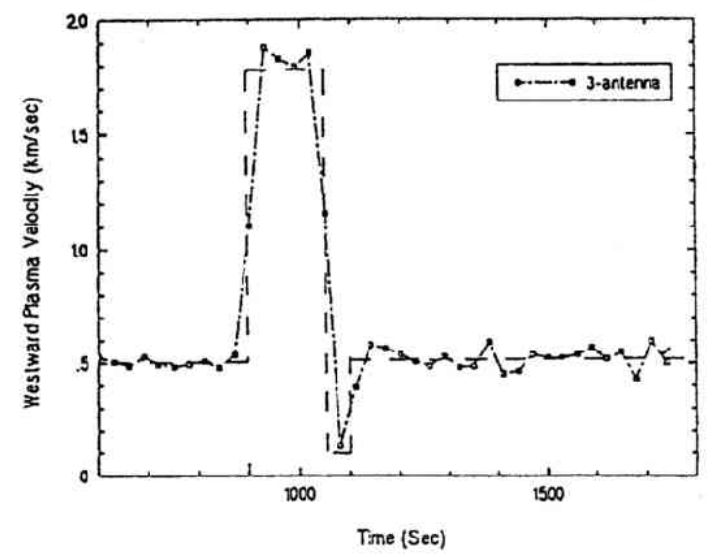

b) Meridional Velocity

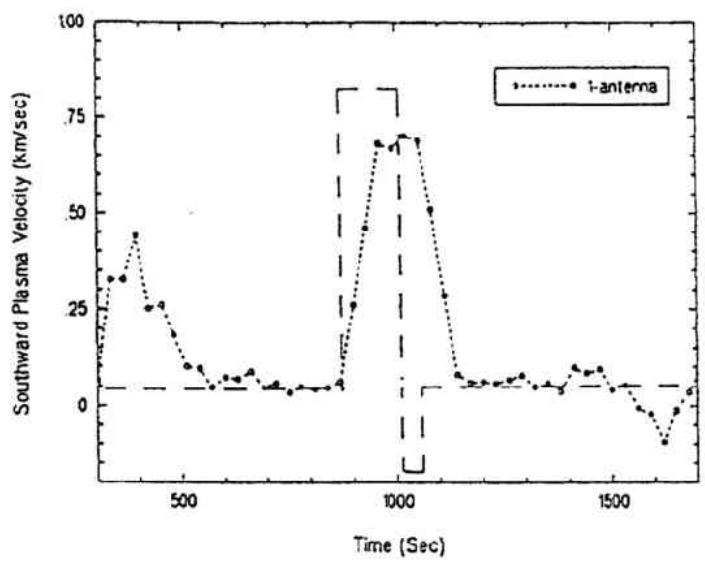

d) Meridional Velocity

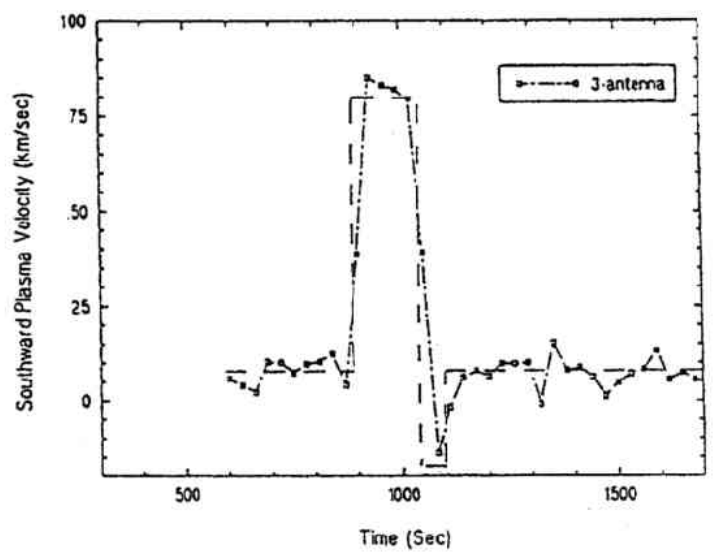

Model Value

Fig. 4. A comparison of the simulated results obtained by a monostatic single-antenna beam-swinging method and a monostatic three-antenna multibeam method when observing an auroral arc drifting over the radar.

For example, in the case where the plasma velocity shows a sharp change at a boundary, a corresponding change will be observed in each component, but at a slightly different time as the boundary passes in turn through the three scattering volumes. Cross-correlation can then be used to determine the time-lags that must bc applied to the three components before they are combined to give an estimate of plasma velocity free from "mixing" errors.

Similarly, in the case of the auroral arc, the sequence of normal velocity/enhanced velocity/reduced velocity/normal velocity will be represented in each of the measured components and once again cross- 
correlation can be used to calculate the optimum time lag to be applied to each component.

In addition to the comparison of (different) velocity components, cross-correlation of scalar parameters such as electron concentration or ion and electron temperature can also be used to confirm the drift velocity of the pattern. For example, frictional heating of $F$-region ions is a reliable indicator of an enhanced electric field strength which is less sensitive to direction than the resolved components of plasma velocity. A time lag of 100 -sec was derived from the cross-correlation of $F$-region ion temperatures observed in a split-beam EISCAT experiment and applied to the velocity components before the vector of plasma velocity was calculated (Lockwood, private communication).

Figures 3(a)-(d) and 4(a)-(d) compare the results obtained when the two methods of observation are simulated. Figures 3(a) and 3(b) give the two components of plasma velocity determined by beamswinging as a sharp boundary drifts through the radar. Comparison with the "true" value shows that although a change in velocity is indicated, large systematic errors occur and Fig. 3(b) shows a case similar to Fig. 2 where one component is actually in the wrong direction. In contrast, the three-beam experiment gives a convincing representation (Figs. 3(c) and 3(d)).

The contrast is even more marked in the case of the arc drifting through the radar. In this case the single-beam measurements seriously underestimate the strength of the enhanced electric field, fail to observe the narrow region of very low electric field, and generate a second band of enhanced electric field which is totally spurious (Figs. 4(a) and 4(b)). Once again the three-antenna measurements faithfully represent the main features (Figs. 4(c) and 4(d)).

A computer simulation is, naturally, a simplified version of the complex reality of auroral activity, but it illustrates the fundamental advantages of a multi-beam facility. Beam-swinging of a single antenna is an invalid way of measuring plasma velocity during any periods of dynamic activity in the auroral zone or polar cap. At best it smoothes out the peaks and troughs in plasma velocity - a serious-enough fault in calculating non-linear functions of electric field such as Joule heating. At worst it generates totally spurious results. In contrast, the multi-antenna method is able to separate space and time, and provided cross-correlation can determine the drift correctly, it gives valid results for the spatial pattern of plasma velocity.

To make an unambiguous determination of the drift velocity three measurements are needed and the long-term plan is to equip the ESR with three antennas. However, many of the advantages of a multi-beam system will already be available if a second antenna is constructed, especially in those circumstances where the approximate drift direction can be assumed or determined from other measurements. In experiments like CP4, CP6 and SP-UK-CONV, EISCAT has already demonstrated the very significant improvement in results obtained in a two-beam mode, and it is hoped that the ESR will enjoy the same improvement as soon as possible.

\section{Measurements of Maximum Sensitivity}

The need to make valid velocity measurements justifies the construction of a multi-antenna system. However, in operating such a system it is important to achieve the maximum sensitivity when splitting transmitter power between two (or three) beams.

From Eqs. (2), (3) and (4) it can be seen that the random error in a velocity measurement made by

a single antenna, under conditions of low $S N R$, is proportional to $\frac{1}{P A \tau^{3 / 2} \sqrt{(n t)}}$.

Now there are two ways to operate a two-antenna radar. The system could be divided completely into two, providing peak power $P / 2$ simultaneously to both radars, and maintaining for each the pulserepetition-rate $n$. In this case the rms error $\delta v$ for a given integration time $t$ will be twice the error for a single antenna transmitting the same total power (or the error will be the same if the integration time $t$ is increased by a factor of 4$)$. 
Alternatively, the full power can be switched to-and-fro between the antennas on alternate pulses. $P$ is maintained but the pulse-repetition-rate for each antenna is now reduced to $n / 2$. In this case the error $\delta v$ is only increased by a factor $\sqrt{2}$ (or the integration time increased by a factor of 2 ).

If, however, the construction of a second antenna is matched by a doubling in peak transmitter power, and if this power $2 P$ is switched between the two antennas, then after the fixed time $t$ the rms error $\delta v$ in the measurements of both antennas will be reduced by $\sqrt{2}$. In other words, if an identical radar is added to phase 1 of the ESR, and the two radars make observations in different directions, then provided the switchyard is designed to allow the combined power to switch between antennas from pulse to pulse* both radars will have a superior performance to that of a single radar operating independently. Under conditions of low signal-to-noise doubling the number of antennas and transmitter modules increases the information flow by a factor of 4 .

Similarly, if a third antenna is constructed and the total peak power increased to $3 P$ then the information flow for low signal-to-noise will increase by a factor of 9 . An outline design for a switchyard which allowed pulse-to-pulse switching between three antennas was proposed by van Eyken and Williams (van Eyken et al., 1989).

In addition, under conditions of very low $S N R$ a multi-antenna system can be used in an alternative mode to provide a single beam with maximum sensitivity. In this case all antennas point in the same direction, sharing the transmitter power with a suitable phase delay so that the signal in the chosen direction is coherent over the combined collecting area.

For example, two parabolic dishes separated by a little more than the dish diameter and looking vertically upwards (or in any direction perpendicular to the baseline separating the dishes) would have an effective collecting area of just under $2 A_{\mathrm{c}}$. When compared with a single antenna and power $P$ the $S N R$ would increase by a factor 4, and at low $S N R$ the random errors would be reduced by a factor of 4 (or the time resolution would improve by a factor of up to 16 ).

It is likely that this mode will be most used in making measurements to the greatest possible height along the field-line. As long as $\lambda$ is greater than the Debye length of the plasma, SNR is proportional to $N /\left(R^{2} b\right)$. In the topside as $R$ increases $N$ drops steadily with height $(\propto c \exp (-z / H))$ and the bandwidth $b$ increases, partly as a result of increasing $T_{\mathrm{i}}$ and partly as a result of a steady fall in average ion mass. Above the $F$-region peak, it therefore becomes extremely difficult to gain extra height but under normal conditions an improvement in $S N R$ by a factor of 4 is equivalent to an increase of about $300-400 \mathrm{~km}$ in the maximum height that can be measured with a given error level after a given integration time.

However, if the two antennas are almost touching when pointing in the plane perpendicular to the baseline, then when they are both pointing away from the vertical in any other direction one antenna will fall in the shadow of the other and in some cases shadowing may even be a problem when the two antennas are pointing in different directions.

Of course, shadowing can be reduced by increasing the separation of the two antennas, but in that case the polar diagram of the single-beam configuration will be strongly modulated by interference fringes with a period determined by the separation.

It follows that in deciding the size and orientation of the baseline for a two-antenna system, a compromise must be struck between the desire to define a effective single-beam for a combined radar and the need to avoid excessive shadowing. It is likely that combined, single-beam operations will be most in demand for observing at maximum height along the magnetic field line, at low elevation in the direction of EISCAT, or at low elevations to higher $L$-values. These observations would be best served by a relatively short baseline approximately perpendicular to the ESR-EISCAT great circle. Such a deploy-

*If alternating codes are used which involve a sequence of, say, 16 coded pulses the switch should occur after one full sequence has been transmitted. Alternating codes require stationarity throughout each sequence to ensure proper cancellation of unwanted cross-products in the correlation of the decoded echoes and so a single sequence should be completed in the minimum possiblc time. The subsequent integration of decoded data over many sequences is less critical and is no different from the normal integration of auto-correlation functions. 
ment would be ideal for most planned experiments, but would give trouble for single-beam or split-beam observations at low elevation to the east or the west.

\section{Flexibility of Operating Modes}

With two (or three) antennas, a sophisticated switchyard that allows total power to be switched between antennas from pulse to pulse or shared between antennas to give in-phase transmission, and sufficient isolation between receiving channels to allow reception by one antenna immediately after transmission from another, the system will have very great flexibility and this will be the third major advantage of a multi-antenna radar at Longyearbyen. Some of the possible modes possible with two (or three) antennas are described in detail in Chapter 4 of a report on a Polar Cap Radar prepared for the Rutherford Appleton Laboratory (Williams, 1990), and in Chapter 6 of the report of the EISCAT PolarCap Radar Working Group (Bjørnå et al., 1991).

The configuration most often used will probably be the split-beam mode with the total power switched between antennas pointing in different directions. This, for example, will be the mode used for measuring plasma velocity above Longyearbyen, with the beams splayed symmetrically away from the magnetic field-line. This mode will be suitable for studying the reversals of plasma velocity at a moving boundary, and the distribution of electric fields near auroral features, as described above. A modified version, with all antennas pointing at different elevations in a single plane in the sunward direction, could be used to study the movement of "patches" over the polar cap.

Alternatively, the split-beams will be directed at low elevation to the south to observe the same scattering volumes as EISCAT looking northwards in split-beam mode. In this configuration it will be possible to determine unambiguously two components of plasma velocity in each scattering volume, and by combining these results the third component can be estimated. This mode will be especially valuable in studying the transient reponse at the dayside magnetopause to changes in the IMF, and plasma flow in the boundary region between the polar cap and the auroral zone will also be investigated.

In a third experiment, the split-beams at low elevation will scan in azimuth to map large-scale convection patterns in the polar cap as well as the distribution of particle precipitation. (It is this third experiment which will be limited if it is decided to use the minimum baseline separating the different antennas.)

The second configuration will be a combined, single-beam radar, used when maximum sensitivity is needed. When the single beam is directed along the field line this mode will be important for measuring ion outflow up to the maximum possible height in the topside. It will therefore be a suitable mode for studying the polar wind and other large upward plasma flows at high altitude near the cusp or in the midnight sector. At low elevation the same configuration looking northward will allow measurements out to the maximum invariant latitude in the polar cap; looking southward it may also provide a suitable mode for joint observations with EISCAT under conditions of very low electron concentration.

A third configuration will direct all transmissions from one antenna, using the other antenna, or antennas, for reception at the same frequency. Reception will then begin as soon as transmission has stopped and this will allow a study of lower heights in the mesosphere and stratosphere than possible when the signals are transmitted and received by the same antenna and a longer recovery time must be allowed after transmission has stopped.

These are only a few obvious ideas of the range of experiments that will be possible with a multiantenna facility. It is certain that once such a system is in operation the range of operational modes will be even greater.

\section{Conclusion}

A single-beam system, as provided by the first phase of the ESR, will provide valuablemeasurements of scalar quantities such as electron concentration and electron and ion temperatures, but such a system 
will be inadequate for a proper study of the dynamics of the polar-cap ionosphere.

At the other extreme, if cost were no barrier, the ideal Svalbard radar would consist of a three-antenna facility at Longyearbyen with receiving antennas at remote sites such as Hornsund or Ny Ålesund. The ideal receiving antenna at a remote site would be a passive, phased array, able to deploy multiple beams covering all three transmitting beams at a number of different heights. (A passive phased array can deploy extra beams without any loss of sensitivity for existing beams: unlike an active phased array where an increase in the number of beams reduces the effective pulse-repetition-rate $n$ for the existing beams and hence increases minimum integration time in direct proportion to the number of beams deployed.)

However, in estimating the scientific return from each addition to the original facility, it is clear that the greatest incremental benefit will come from the construction of the second antenna at Longyearbyen and this should be given the highest priority.

I wish to thank the Solar-Environment Laboratory at Nagoya University Japan and the EISCAT Scientific Association for organising the Japan-EISCAT Symposium on the Polar Ionosphere, and the Particle Physics and Astronomy Research Council of the UK for enabling me to attend.

\section{REFERENCES}

Bauer, P., P. Waldteufel, and C. Vialle, Radio Sci., 9, 77-83, 1974.

Bjørnå, N., B. Hultqvist, W. Kofman, J. Rottger, K. Schlegel, T. Turunen, and D. M. Willis, The EISCAT Svalbard Radar, Rutherford Appleton Laboratory, UK, 1991.

Cowley, S. W. H., A. P. van Eyken, E. C. Thomas, P. J. S. Williams, and D. M. Willis, Studies of the cusp and auroral zone with incoherent scatter radar: the scientific and technical case for a polar cap radar, J. Atmos. Terr. Phys., 52, 645-663, 1990.

Etemadi, A., S. W. H. Cowley, and M. Lockwood, The effecty of rapid changes in ionospheric flow on velocity vectors deduced from radar beam-swinging experiments, J. Atmos. Terr. Phys., 51, 125-138, 1989.

van Eyken, A. P., H. Rishbeth, D. M. Willis, and S. W. H. Cowley, Initial EISCAT observations of plasma convection at high latitudes, J. Atmos. Terr. Phys., 46, 635-641, 1984.

van Eyken, A. P., E. C.Thomas, P. J. S. Williams, and D. M. Willis, The Polar Cap Radar, Case to the Astronomy and Planetary Science Board of the SERC, UK, 1989.

Farmer, A. D., G. O. L. Jones, P. J. S. Williams, R. J. Moffett, A. Samson, R. B. Horne, M. J. Rycroft, M. Lester, and I. W. McCrea, The ionosphere south of EISCAT: measurements by the UK-South programme, J. Atmos. Terr. Phys., 52, 735-747, 1990.

Jones, G. O. L., K. J. Winser, and P. J. S. Williams, Measurements of plasma velocity at different heights along a magnetic field line, J. Atmos. Terr. Phys., 48, 887-892, 1986.

Lanchester, B. S., K. Kaila, and I. W. McCrea, Relationship between large horizontal electric fields and auroral arc elements, $J$. Geophys. Res., 1995 (in press).

Lewis, R. V., P. J. S. Williams, T. S. Virdi, T. K. Yeoman, and M. Lester, EISCAT measurements of bursts in plasma velocity during substorm activity, Proc. ESA Symposium on Substorms, Kiruna, 125-129, 1992.

Lewis, R. V., P. J. S. Williams, G. O. L. Jones, H. J. Opgenoorth, and M. A. L. Persson, Electrodynamics of a drifting auroral arc, Ann. Geophys., 12, 478-480, 1994.

Lockwood, M., On flow reversal boundaries and transpolar voltage in average models of high-latitude convection, Planet. Space Sci., 39, 397-409, 1991a.

Lockwood, M., The excitation of ionospheric convection, J. Atmos. Terr. Phys., 53, 177-199, 1991b.

Luhr, H. and K. Schlegel, Combined measurements of EISCAT and thge EISCAT Magnetometer Cross to study omega bands, J. Geophys. Res., 99, 8951-8959, 1994.

Oliver, W. L., S. Fukao, T. Sato, T. Tsuda, S. Kato, I. Kimura, A. Ito, T. Saryou, and T. Araki, Ionospheric incoherent scatter measurements with the MU radar: Observations of $F$-region electrodynamics, J. Geomag. Geoelectr., 40, 963-985, 1988.

Opgenoorth, H. J., I. Häggström, P. J. S. Williams, and G. O. L. Jones, Regions of strongly enhanced perpendicular electric fields adjacent to auroral arcs, J. Atmos. Terr. Phys., 52, 449-458, 1990.

Rishbeth, H., G. N. Taylor, and P. J. S. Williams, Multi-static incoherent scatter measurements of ionospheric drifts, Nature, Lond., 242, 109-111, 1973.

Rishbeth, H. and P. J. S. Williams, The EISCAT ionospheric radar: the system and its early results, Q. J. R. astr. Soc., 26, 478$512,1985$.

Williams, P. J. S., Report on an Incoherent-Scatter Radar on Svalbard, 45 pp., University of Wales Aberystwyth, 1990.

Williams, P. J. S., G. O. L. Jones, and A. R. Jain, Methods of measuring plasma velocity with EISCAT, J. Atmos. Terr. Phys., 46, 521-530, 1984.

Williams, P. J. S., T. S. Virdi, S. W. H. Cowley, and M. Lester, Short-lived bursts of plasma velocity in the auroral zone: 
observational evidence from radar measurements, J. Atmos. Terr. Phys., 52, 421-430, 1990a.

Williams, P. J. S., G. O. L. Jones, B. Jones, I. Häggström, and H. J. Opgenoorth, High resolution measurements of the magnetospheric electric field, J. Atmos. Terr. Phys., 52, 439-448, 1990 b.

Williams, P. J. S., R. V. Lewis, T. S. Virdi, M. Lester, and E. Nielsen, Plasma flow bursts in the auroral clectrojets, Ann. Geophys., 10, 835-848, 1992.

Williams, P. J. S., T. S. Virdi, G. O. L. Jones, and A. Huuskonen, A comparison of three methods of measuring tidal oscillations in the lower thermosphere using EISCAT common programmes, J. Atmos. Terr. Phys., 56, 1347-1359, 1994. 